Also, the determinant of equations (7)-(9) vanishes, as may be seen at once from the fact that (9) may be obtained by subtracting $\beta^{\prime}$ times (7) from $\beta$ times (8). Hence* we have

THEOREM II. The parameters of the points of contact of the three pairs of tangents that can be drawn to the $R^{3}$ from three collinear points of the $R^{3}$ are harmonic to the same quadratic, or form a set in involution.

Another result which may be derived as a corollary of Theorem I we shall state as

Theorem III. Lines on a point $P$ of an $R^{3}$ cut the $R^{3}$ in pairs of residual points whose parameters are harmonic to the parameters of the points of contact of the two additional tangents drawn to $R^{3}$ from $P$.

Although Theorem III may be regarded a corollary of Theorem I, it may be established independently. Thus: Let $P\left(d_{0}, d_{1}, d_{2}\right)$ be the point and $(\kappa x) \equiv \kappa_{0} x_{0}+\kappa_{1} x_{1}+\kappa_{2} x_{2}=0$ any line on $P$. Then $(\kappa d)=0$. The parameters of the residual points cut out of (1) by $(\kappa x)=0$ are the roots of

$$
(\kappa a) t^{2}+3(\kappa b) t+3(\kappa c)=0
$$

and (10) is apolar to (8), for

$$
3(\kappa c) \beta+3(\kappa a) \alpha^{\prime}-3(\kappa b) \beta^{\prime}=0,
$$

as may be shown from (3) and the fact that $(\kappa d)=0$.

Pennsylvania State College, March, 1917.

\title{
EXAMPLES OF A REMARKABLE CLASS OF SERIES.
}

BY PROFESSOR R. D. CARMICHAEL.

(Read before the American Mathematical Society, April 28, 1917.)

Two-Fold and One-Fold Expression of the Properties of Functions.

1. In the development of analysis during the past generation it has frequently happened that functions have arisen which are analytic in a sector of the complex plane and in

* Salmon, Higher Algebra, fourth edition, p. 180. 
that sector have a determinate asymptotic representation (in the sense of Poincaré) by means of a diverging power series. The first explicit appearance of these functions in a general way was in two memoirs by Poincaré* in 1885 and 1886 .

The importance of these functions was at once put in evidence by Poincare's association of them with the irregular singular point at infinity of the linear homogeneous differential equation

$$
P_{0} \frac{d^{n} y}{d x^{n}}+P_{1} \frac{d^{n-1} y}{d x^{n-1}}+\cdots+P_{n} y=0,
$$

in which the coefficients $P_{0}, P_{1}, \cdots, P_{n}$ are polynomials in $x$ subject to the condition that their degrees do not constantly decrease as one passes in order from the first to the last polynomial in the sequence. Poincaré (Acta Mathematica, loc. cit.) has in fact shown that a solution $y(x)$ of such an equation is in general asymptotic to an expression of the form

$$
x^{\mu} e^{Q(x)}\left(1+\frac{a_{1}}{x}+\frac{a_{2}}{x^{2}}+\cdots\right),
$$

where $\mu$ is a constant and $Q(x)$ is a polynomial in $x$, the asymptotic representation being valid for $x$ approaching infinity in an appropriate direction.

In other important investigations functions of the same character have often made their appearance, particularly in the theory of differential and difference equations. In these investigations it has been usual to represent the asymptotic character of such a function by means of a diverging power series and to find in addition another form through which to put in evidence the analytic character of the function in the sector. In this connection use has frequently been made of the integral on which the Laplace transformation is based or of certain generalizations of it. In other cases it has been found necessary to resort to expansions of various types, particularly of those which arise through use of the method of successive approximation. These procedures do not always lead to suitably convenient representations of the functions in consideration; in particular, the expansions last mentioned are often complicated in character.

* Amer. Jour. of Math., vol. 7 (1885), pp. 203-258; Acta Mathematica, vol. 8 (1886), pp. 295-344. Particular instances of such functions appeared earlier, notably in the work of Stirling and Cauchy (see Borel's Séries divergentes, p. 30). 
2. Obviously, it is desirable, if possible, to have a single expansion of such character that it is capable of exhibiting the asymptotic properties of the function near infinity and of yielding at the same time a convenient and workable representation of it in the finite plane or in a significant portion of the finite plane. It is hardly to be expected that any single class of series will afford a form of such a tool best suited to all situations; but it may very well be (and we are about to exhibit a class of series in which it is) true that certain classes of functions of great importance and very frequently recurring in practice are capable of representation in one or another sort of series all of which belong to a single type and possess a unitary theory.

Indeed it has recently appeared that factorial series and certain immediate generalizations of them serve just such a purpose to a remarkable degree. Nörlund* has shown that functions which are characterized by being analytic in a halfplane and having an asymptotic character of a certain broad type are expansible in convergent factorial series (of a slightly generalized form) and that these series do in fact readily show the asymptotic character of the functions represented by them. In connection with a range of ideas differing in some essential respects from those of Nörlund it was shown earlier by Watson $\dagger$ that a certain form of factorial series furnished a sufficient means of representing a very important and wide-reaching class of functions. About the same time Hornł employed another generalization of factorial series in obtaining suitably convenient representations of solutions of linear differential equations in the neighborhood of an irregular singular point. Nörlund§ has likewise employed factorial series in his fundamental investigation of the solutions of difference equations.

3. In a recent memoir|| I have pointed out that factorial series whose fundamental importance has been made manifest in these several ways are but an instance of a large class of series of simple properties. It turns out that the latter are

* Acta Mathematica, vol. 37 (1914), pp. 327-387.

† Rendiconti del Circolo Matematico di Palermo, vol. 34 (1912), pp. 41-88.

$\$$ Math. Annalen, vol. 71 (1912), pp. 510-532. $177-216$.

$\$$ Rendiconti del Circolo Matematico di Palermo, vol. 35 (1913), pp.

II Transactions Amer. Math. Soc., vol. 17 (1916), pp. 207-232. See also a forthcoming paper in the Amer. Jour. of Math. 
suitable for the representation of functions which are defined throughout a sector and have certain types of singularities at infinity. Moreover these series afford the requisite information concerning the asymptotic character of the functions represented by them.

These series are of the form

$$
\Omega(x)=\sum_{n=0}^{\infty} c_{n} \frac{g(x+n)}{g(x)},
$$

where $g(x)$ is a given function of $x$ and the coefficients $c_{n}$ are independent of $x$. More precise definitions are to be found in the next sections. As will be seen from these definitions, the fundamental characteristic of $g(x)$ is its asymptotic behavior.

A detailed study of the more important series included in this class and especially of their suitability for the representation of certain types of functions is desirable and leads to important consequences in the theory of functions. From these latter the close connection of the series $\Omega(x)$ with the function-theoretic considerations mentioned in the preceding sections will become apparent.

The principal object of the present paper is to show how several important series in the literature are included as special cases of the series $\Omega(x)$ and the generalization $T(x)$ of it introduced in section 6 and to exhibit certain other interesting examples. I hope that attention may thus be directed to the importance of this entire class of series. I add also a brief discussion of the region of convergence of the new series $T(x)$.

$$
\text { Definition of the Series } \Omega(x) \text { and } T(x) \text {. }
$$

4. The class of series $\Omega(x)$ is determined primarily by the properties of the basic function $g(x)$ through which the series are defined. Accordingly it is desirable to begin with a statement of the properties of suitable functions $g(x)$.

In every case we shall confine our attention to a defined portion of the complex plane and usually to a sector formed by two rays from zero to infinity and containing in its interior the positive axis of reals. In such a portion of the plane (appropriately defined) we assume that $g(x)$ is single-valued and also that it is analytic when $|x|$ is sufficiently large. Moreover it is to have the asymptotic properties expressed in the formula 


$$
g(x) \sim x^{P(x)} e^{Q(x)}\left(1+\frac{a_{1}}{x}+\frac{a_{2}}{x^{2}}+\cdots\right),
$$

in which $P(x)$ and $Q(x)$ are polynomials which we write in the form

$$
\begin{array}{ll}
P(x)=\mu_{0}+\mu_{1} x+\mu_{2} x^{2}+\cdots+\mu_{k} x^{k} & \left(\mu_{k} \neq 0 \text { if } k>0\right), \\
Q(x)=\alpha_{0}+\alpha_{1} x+\alpha_{2} x^{2}+\cdots+\alpha_{m} x^{m} & \left(\alpha_{m} \neq 0 \text { if } m>0\right) .
\end{array}
$$

By $r^{t}$ we mean $e^{t \log r}$, where the principal determination of $\log r$ is taken.

In case $k=0$ we assume that $m>1$ in order to avoid a case which is unimportant so far as our present objects are concerned.

The asymptotic relation (1) is to be understood as an abbreviation for the infinite sequence of limits

(2) $\lim x^{s}\left(g(x) x^{-P(x)} e^{-Q(x)}-\left(1+\frac{a_{1}}{x}+\frac{a_{2}}{x^{2}}+\cdots+\frac{a_{s}}{x^{s}}\right)\right)$

$$
=0 \quad(s=0,1,2, \cdots) \text {, }
$$

the limits being taken for $x$ approaching infinity in a sector including the positive axis of reals in its interior.

5. By means of any function $g(x)$ possessing the properties just indicated we introduce the series $\Omega(x)$ defined by the relation

$$
\Omega(x)=\sum_{n=0}^{\infty} c_{n} \frac{g(x+n)}{g(x)},
$$

where the coefficients $c_{0}, c_{1}, c_{2}, \cdots$ are independent of $x$.

In case $g(x+n)$ and $g(x)$ have common zeros we shall understand that $g(x+n) / g(x)$ denotes the function obtained when the numerator and denominator of this fraction are divided by an entire function (preferably a polynomial when possible) having for its zeros the common zeros of $g(x+n)$ and $g(x)$.

A value of $x$ for which any one of the functions $g(x)$, $g(x+1) / g(x), g(x+2) / g(x), \cdots$ has a singularity will be called an exceptional value or an exceptional point for the series $\Omega(x)$. Other values of $x$ will be called non-exceptional or ordinary.

In order to avoid cases without value for our purposes we shall assume that $g(x)$ is of such character that there is a 
(two-dimensional) region in the $x$-plane containing no exceptional points for the series.

6. Let us suppose that we have a finite number $\nu+1$ of series $\Omega(x)$ which, with $x$ replaced by $x^{t}$ where $t$ is a positive integer, we write in the form

$$
\Omega_{i}\left(x^{t}\right)=\sum_{n=0}^{\infty} c_{n i} \frac{g\left(x^{t}+n\right)}{g\left(x^{t}\right)} \quad(i=0,1,2, \cdots, \nu) .
$$

Let us multiply $\Omega_{i}\left(x^{t}\right)$ by $1 / x^{i}(i=0,1,2, \cdots, \nu)$ and add the resulting series term by term. We are thus led to a new series

$$
T_{\nu t}(x)=\sum_{n=0}^{\infty} \mid P_{n \nu}(x) \frac{g\left(x^{t}+n\right)}{g\left(x^{t}\right)},
$$

where $P_{n \nu}(x)$ is a polynomial of degree $\nu$ in $1 / x$, namely,

$$
P_{n \nu}(x)=c_{n 0}+\frac{c_{n 1}}{x}+\frac{c_{n 2}}{x^{2}}+\cdots+\frac{c_{n \nu}}{x^{\nu}} .
$$

Exceptional points and ordinary points are here defined as in section 5 , the point $x=0$ being now added to the set of exceptional points.

These series $T(x)$ are here introduced for the first time. It is clear that they have a much greater flexibility than series $\Omega(x)$ in adapting themselves to the necessities of representing a function which is arbitrary save as to general properties.

7. The definitions of series $\Omega(x)$ and $T(x)$ might be extended to the case in which (1) is replaced by the relation

$$
g(x) \sim x^{P(x)} e^{Q(x)}\left(1+\frac{a_{1}}{x^{1 / p}}+\frac{a_{2}}{x^{2 / p}}+\cdots\right)
$$

and $P(x)$ and $Q(x)$ are polynomials either in $x$ or more generally in $x^{1 / p}$. Suitable functions $g(x)$ for use in defining these more general series arise in situations analogous to those mentioned in sections 8 and 9 below. The theory of these more general series $\Omega(x)$ and $T(x)$ will not be treated in this paper.

Convenient Sources of Suitable Functions $g(x)$.

8. It is obvious that there exists a large class of series $\Omega(x)$ in accordance with the foregoing definition; for one has the subclass of functions $g(x)$ for which the series in the second 
member of (1) is convergent when $|x|$ is sufficiently large and in particular that in which the series terminates, the case in which it consists of the first term alone being of especial interest (as we shall see in section 11).

That there also exist other important basic functions $g(x)$ may be readily seen. In fact, as we saw in section 1, solutions $y(x)$ of linear homogeneous differential equations of a certain broad type are found to possess in general the asymptotic representation

$$
y(x) \sim x^{\mu} e^{Q(x)}\left(1+\frac{a_{1}}{x}+\frac{a_{2}}{x^{2}}+\cdots\right)
$$

valid for $x$ approaching infinity in an appropriate direction, $Q(x)$ being a polynomial in $x$ and $\mu, a_{1}, a_{2}, \cdots$ being constants. Hence a constant $c$ exists such that $y(c x)$ is a function having the property specified above for $g(x)$.

9 . Another class of functions $g(x)$ is afforded by the theory of difference equations. These functions are usually more effective than those defined by differential equations because the sequence of functions

$$
\frac{g(x+1)}{g(x)}, \quad \frac{g(x+2)}{g(\dot{x})}, \frac{g(x+3)}{g(x)}, \ldots
$$

has in this case very simple properties of interrelation among its elements, more convenient for most purposes than in the case of functions defined by differential equations. A theorem asserting the existence of these functions may be stated as follows:*

Let us consider the linear homogeneous difference equation of order $n$

$$
F(x+n)+a_{1}(x) F(x+n-1)+\cdots+a_{n}(x) F(x)=0,
$$

in which the coefficients $a_{k}(x)$ have the property that $x^{-\mu k} a_{k}(x)$ is analytic at infinity for every value of $k$ from 1 to $n, \mu$ being a constant. By $e^{\mu k} a_{k 0}$ we denote the value of $x^{-\mu k} a_{k}(x)$ at infinity. We assume that the quantities $a_{k 0}$ are such that the characteristic algebraic equation

$$
\alpha^{n}+a_{10} \alpha^{n-1}+\cdots+a_{n 0}=0
$$

has its roots $\alpha_{1}, \alpha_{2}, \cdots, \alpha_{n}$ different from each other and from

\footnotetext{
* See Amer. Jour. of Math., vol. 38 (1916), pp. 185-220, and the papers there referred to.
} 
zero. In this case the difference equation has $n$ formal (and in general divergent) power series solutions of the form

$$
F_{i}(x)=x^{\mu x} \alpha_{i}^{x} x^{\mu_{i}}\left(1+\frac{c_{i 1}}{x}+\frac{c_{i 2}}{x^{2}}+\cdots\right)(i=1,2, \cdots, n),
$$

where $\mu_{i}, c_{i 1}, c_{i 2}, \cdots$ are constants which may be reckoned out directly by substituting the expansion in the difference equation and equating like powers of $x$ in the result.

Then the difference equation has a fundamental system of solutions

$$
F_{1}(x), F_{2}(x), \cdots, F_{n}(x)
$$

possessing the following properties:

(1) Each function in the system is analytic throughout the finite plane except at the singularities $\alpha$ of the functions

$$
\frac{1}{a_{n}(x)}, \quad \frac{a_{1}(x)}{a_{n}(x)}, \quad \frac{a_{2}(x)}{a_{n}(x)}, \cdots, \frac{a_{n-1}(x)}{a_{n}(x)}
$$

and the points $\alpha-i$ where $i$ is a positive integer.

(2) The functions $F_{1}(x), F_{2}(x), \cdots, F_{n}(x)$ have with respect to $x$ the asymptotic representation

(5) $\quad F_{i}(x) \sim x^{\mu x} \alpha_{i}^{x} x^{\mu_{i}}\left(1+\frac{c_{i 1}}{x}+\frac{c_{i 2}}{x^{2}}+\cdots\right)$

$(i=1,2, \cdots, n)$,

this representation being valid for $x$ approaching infinity in any sector $V$ formed by two rays from zero to infinity with arguments less than $\pi / 2$ in absolute value.

Very useful particular basic functions $g(x)$ for series $\Omega(x)$ and $T(x)$ are afforded directly by the foregoing functions $F_{i}(x)$, especially in the case when $\mu$ is a negative integer. If $\mu$ is not a negative integer then a suitable and convenient function $g(x)$ in place of $F_{i}(x)$ is given by

$$
g(x)=F_{i}(x)[\Gamma(x)]^{-\mu-k},
$$

where $k$ is a positive integer and $\Gamma(x)$ denotes the gamma function of Euler. For the case when $k$ is unity these functions are especially useful. Moreover, any one of many expressions similar to the foregoing readily serves the same purpose.

The case when the difference equation is of the first order 
deserves special mention on account of the elegance of many of the series arising through functions $g(x)$ satisfying equations of the form

$$
g(x+1)=R(x) g(x),
$$

where $R(x)$ is a rational function of $x$. See sections $12-17$ below.

10. Series $\Omega(x)$ and $T(x)$ give rise to what appears to be the fundamental expansion problem in the theory of difference equations; or, more exactly, they give rise to a general problem which includes this one as a special case and also a corresponding one in the theory of differential equations. The general problem is far more comprehensive than either of these special cases.

The total class of series $\Omega(x)$ and $T(x)$ is very large owing to the paucity of restrictions which it is necessary to put upon the basic functions $g(x)$. So far as I can see now, however, it appears probable that the functions $g(x)$ afforded by the solutions of linear homogeneous difference equations give rise to the most flexible series in the total class and are thus likely to prove themselves of the greatest permanent use in developing the theory of functions. It is for this reason that I venture to suggest tentatively that the series treated in this paper give rise to the fundamental expansion problem in the theory of difference equations.

It is clear, however, that this expansion problem is not analogous to that having to do with orthogonal and biorthogonal functions in the theory of differential equations. Owing to the fact that many common properties are possessed by difference and differential equations it is natural to expect in the theory of the former the analogue of expansions in fundamental functions arising in the theory of the latter. That such a theory could be developed is highly probable; but it is not at present in existence. It seems improbable, however, that the expansions so obtainable are commensurate in importance with expansions in the form of our series $\Omega(x)$ and $T(x)$.

Special Cases of Series $\Omega(x)$ and $T(x)$ in the Literature.

11. Let us consider the special case in which $g(x)$ has the value

$$
g(x)=e^{\sigma x^{2}} .
$$


Then $\Omega(x)$ is readily reduced to the form

$$
\Omega(x)=\sum_{n=0}^{\infty} c_{n} g(n) e^{2 \sigma n x} .
$$

If we make the substitution $z=e^{2 \sigma x}$ the series $\Omega(x)$ is transformed into the power series

$$
\sum_{n=0}^{\infty} c_{n} g(n) z^{n}
$$

Hence among the special cases of series $\Omega(x)$ is to be found the foregoing elementary transform of a general power series.

12. It is well known that the function $\Gamma(x)$ has the asymptotic character

$$
\Gamma(x) \sim x^{x-\frac{1}{2}} e^{-x} \sqrt{2 \pi}\left(1+\frac{1}{12 x}+\cdots\right) .
$$

Hence for $g(x)$ we may take the special value

$$
g(x)=\frac{1}{\Gamma(x)} \sim x^{-x+\frac{1}{2}} e^{x}\left(a_{0}+\frac{a_{1}}{x}+\cdots\right) .
$$

Then we have

$$
\frac{g(x+n)}{g(x)}=\frac{\Gamma(x)}{\Gamma(x+n)}=\frac{1}{x(x+1)(x+2) \cdots(x+n-1)} .
$$

Hence for this case our $\Omega$-series takes the form

$$
\Omega(x)=c_{0}+\sum_{n=1}^{\infty} \frac{c_{n}}{x(x+1)(x+2) \cdots(x+n-1)} .
$$

This is the well-known factorial series. The so-called series of binomial coefficients arises similarly by taking for $g(x)$ the value

whence we have

$$
g(x)=\Gamma(x),
$$

$$
\Omega(x)=c_{0}+\sum_{n=1}^{\infty} c_{n} x(x+1) \cdots(x+n-1) .
$$

13. Another interesting example of our series $\Omega(x)$ is obtained by taking for $g(x)$ the value

$$
g(x)=\frac{1}{a^{x} \Gamma(x+b / a)} \sim x^{-x+\mu} e^{a+\beta x}\left(1+\frac{a_{1}}{x}+\cdots\right),
$$


where $a$ and $b$ are constants and $a \neq 0$. In this case the $\Omega$-series takes the form

$$
\Omega(x)=c_{0}+\sum_{n=1}^{\infty} \frac{c_{n}}{(a x+b)(a x+b+a) \cdots(a x+b+(n-1) a)} .
$$

Let us now give to $b$ the value 0 and replace $a x$ by $z$. Then if we put $c_{0}=0$ the foregoing series takes the form

$$
S(z)=\sum_{n=1}^{\infty} \frac{c_{n}}{z(z+a)(z+2 a) \cdots(z+(n-1) a)} .
$$

This series plays the leading rôle in Nörlund's fundamental paper on factorial series referred to in section 2.

Concerning series $S(z)$ Nörlund proposes a fundamental problem in the following manner: What is the class of functions which admit an expansion of the form $S(z)$ for appropriately chosen real values of $a$ ? To this question he finds a very interesting answer. He shows that the class of functions is the same as that which gives rise to power series of the form

$$
\frac{A_{1}}{z}+\frac{A_{2}}{z^{2}}+\frac{A_{3}}{z^{3}}+\cdots
$$

which may be divergent but which are absolutely and uniformly summable by the exponential method of Borel. An infinite number of functions may give rise to the same power series; but it is the function which Borel assigns to this series as its generalized sum that admits an expansion of the form $S(z)$.

Thus a special class of the $\Omega$-series is in close relation with one of the most interesting questions of analysis, namely, the question as to what we shall mean by the sum of a divergent series.

Moreover the function defined by the series $S(z)$ is asymptotic to the power series of which it is the Borel sum; and another interesting point of connection for series $\Omega(x)$ thus emerges.

In later memoirs I intend to exhibit these and other properties of the series $S(z)$ in their place as special cases of properties possessed by the $\Omega$-series in general.

14. Again, if we put $M z$ for $x$ and $\alpha+1$ for $b / a$ in the $\Omega$ - 
series of the preceding section, we obtain a new series which is readily reduced to the form

$$
\bar{S}(z)=b_{0}+\sum_{n=1}^{\infty} \frac{b_{n}}{(M z+\alpha+1)(M z+\alpha+2) \cdots(M z+\alpha+n)} .
$$

This series plays the leading rôle in Watson's fundamental paper referred to in section 2. Watson shows that most of the ordinary functions of analysis (which possess asymptotic expansions) are capable of convergent representations in the form of series $\bar{S}(z)$. This statement is justified by three leading results which are stated by him as Theorems I, II, III. Through such results one sees from another point of view the importance of our series $\Omega(x)$.

15. In a suggestive paper on the solutions of homogeneous linear differential equations in the neighborhood of the irregular point infinity Horn (loc. cit.) has made use of a series which he writes in the form

$$
\begin{aligned}
A_{0} & +\sum_{n=1}^{\infty}\left(A_{n}+B_{n} x\right) \\
& \times \frac{(\lambda+1)(\lambda+2) \cdots(\lambda+n)}{\left(x^{2} / k+\lambda+1\right)\left(x^{2} / k+\lambda+2\right) \cdots\left(x^{2} / k+\lambda+n\right)} .
\end{aligned}
$$

He treats the equation

$$
P_{0} \frac{d^{2} y}{d x^{2}}+P_{1} \frac{d y}{d x}+P_{2} y=0,
$$

in which the coefficients $P_{0}, P_{1}, P_{2}$ are polynomials in $x$ of degrees $m, m+1, m+2$ such that the characteristic algebraic equation for the point infinity of the differential equation has its roots distinct. For such an equation the point infinity is irregular of rank 2. Horn shows that the solutions of such an equation can be represented by means of convergent series (8) and he points out that the results are capable of generalization to equations of any order and with any rank of irregular point at infinity.

It is thus apparent that series (8) are of great importance in the theory of differential equations.

We shall now exhibit series (8) as directly dependent on a special case of our series $T(x)$. For this purpose we choose for $g(x)$ the value 


$$
g(x)=\frac{1}{\Gamma(x+\lambda+1)},
$$

so that we have

$$
\frac{g(x+n)}{g(x)}=\frac{1}{(x+\lambda+1)(x+\lambda+2) \cdots(x+\lambda+n)} .
$$

Thence we may write

$$
\begin{gathered}
\Omega_{1}\left(x^{2}\right)=c_{0}+\sum_{n=1}^{\infty} \frac{c_{n}}{\left(x^{2}+\lambda+1\right) \cdots\left(x^{2}+\lambda+n\right)}, \\
\Omega_{2}\left(x^{2}\right)=\sum_{n=1}^{\infty} \frac{d_{n}}{\left(x^{2}+\lambda+1\right) \cdots\left(x^{2}+\lambda+n\right)} .
\end{gathered}
$$

Multiplying the first of these series by 1 and the second by $x$ and adding, we have

$$
x T(x)=c_{0}+\sum_{n=1}^{\infty} \frac{c_{n}+d_{n} x}{\left(x^{2}+\lambda+1\right) \cdots\left(x^{2}+\lambda+n\right)},
$$

where $T(x)$ denotes an instance of the series $T_{\nu t}(x)$ defined in section 6 . If we now replace $x$ by $x / \sqrt{k}$ it is clear that we have a series of the form (8).

Thus we have exhibited several series of analysis as special cases of our general class of $\Omega$-series; in this way we have tentatively shown the importance of the latter series.

\section{Some Other Special Cases of Interest.}

16. Let us consider the first order difference equation

$$
g(x+1)=R(x) g(x) \equiv a \frac{\left(x-\alpha_{1}\right)\left(x-\alpha_{2}\right) \cdots\left(x-\alpha_{m}\right)}{\left(x-\beta_{1}\right)\left(x-\beta_{2}\right) \cdots\left(x-\beta_{k}\right)} g(x),
$$

in which the order $m-k$ of $R(x)$ at infinity is different from zero. This equation obviously has a solution of the form

$$
g(x)=a^{x} \frac{\Gamma\left(x-\alpha_{1}\right) \cdots \Gamma\left(x-\alpha_{m}\right)}{\Gamma\left(x-\beta_{1}\right) \cdots \Gamma\left(x-\beta_{k}\right)} .
$$

From the asymptotic representation of $\Gamma(x)$, quoted above, it follows readily that the foregoing function $g(x)$ has an asymptotic representation of the form 


$$
g(x) \sim x^{\mu x+v} e^{a x+\beta}\left(1+\frac{a_{1}}{x}+\frac{a_{2}}{x^{2}}+\cdots\right), \quad \mu=m-k .
$$

Hence the function $g(x)$ is suitable for use in defining $\Omega$-series. It is clear that we have

$$
\begin{aligned}
\frac{g(x+n)}{g(x)}= & \frac{g(x+1)}{g(x)} \cdot \frac{g(x+2)}{g(x+1)} \cdot \cdots \cdot \frac{g(x+n)}{g(x+n-1)} \\
& =R(x) R(x+1) \cdots R(x+n-1) .
\end{aligned}
$$

Hence our series $\Omega(x)$ in this case takes the form

$$
\Omega(x)=c_{0}+\sum_{n=1}^{\infty} c_{n} R(x) R(x+1) \cdots R(x+n-1) .
$$

This form of series $\Omega(x)$ essentially includes as special cases the three types of series mentioned in sections 12-14. It affords a highly interesting class of expansions in rational functions, particularly when $R(x)$ vanishes to the first order at infinity, as I intend to show in a future paper.

Let us consider the special case in which $R(x)$ vanishes at infinity to the integral order $s+1$. Then $\mu=-s-1$. For this case we have a particularly useful class of series $T(x)$, namely,

$T(x)=c_{00}+\frac{c_{01}}{x}+\cdots+\frac{c_{0 s}}{x^{8}}$

$+\sum_{n=1}^{\infty}\left(c_{n 0}+\frac{c_{n 1}}{x}+\cdots+\frac{c_{n s}}{x^{s}}\right) R(x) R(x+1) \cdots R(x+n-1)$.

It is not difficult to show that this series may be formally transformed into a descending power series

$$
\beta_{0}+\frac{\beta_{1}}{x}+\frac{\beta_{2}}{x^{2}}+\cdots
$$

and conversely that any such power series may be formally transformed into a series $T(x)$ depending on a given $R(x)$ vanishing at infinity to the order $s+1$. The transformation is unique in each direction. Moreover the power series may diverge for all values of $x$ while the $T$-series has a halfplane of convergence. It is in connection with such facts that these $T$-series exhibit their great importance, as I shall show in detail in a later paper. 
17. A certain subclass of the functions $g(x)$ employed in the preceding section is of special interest on account of their applications. This subclass consists of those functions $g(x)$ in which $g(x) / g(x+1)$ is a rational function of $x$ and $g(x) / g(x+n)$ is a polynomial in $x$ provided that $n$ is sufficiently large. A useful more restricted class is that in which this polynomial has non-negative real coefficients. We shall now exhibit a few such functions $g(x)$.

We take first a case already treated, namely, that in which

$$
g(x)=\frac{1}{a^{x} \Gamma(x+b / a)}, \quad a \neq 0 .
$$

Here $R(x)$ has the value $1 /(a x+b)$. Hence $g(x) / g(x+n)$ is a polynomial in $x$ of degree $n$. Moreover, its coefficients are non-negative and real if $a$ is positive and $b$ is non-negative and real.

Again, we may take

$$
g(x)=\frac{\Gamma(x+1)}{\Gamma(x) \Gamma(x+3)} .
$$

Here it is easy to see that $g(x) / g(x+n)$ is a polynomial with non-negative real coefficients provided that $n$ is sufficiently large, in fact, provided that $n$ is greater than 2 .

In a similar way one may treat the function

$$
g(x)=\frac{\Gamma(x+1) \Gamma(x+3)}{\Gamma(x) \Gamma(x+2) \Gamma(x+4)}
$$

and with a like result.

In the foregoing cases $R(x)$ vanishes to the first order at infinity. In the following it vanishes to the second order:

$$
\begin{aligned}
g(x) & =\frac{\Gamma(x+1)}{\Gamma(x) \Gamma(x+3) \Gamma(x+4)}, \\
g(x) & =\frac{\Gamma(x+1) \Gamma(x+3)}{\Gamma(x) \Gamma(x+2) \Gamma(x+4) \Gamma(x+5)} .
\end{aligned}
$$

It is obvious in what way one may form other functions $g(x)$ so that $R(x)$ vanishes at infinity to any desired order while $g(x) / g(x+n)$ has the desired polynomial character.

18. If we take for $g(x)$ a function of the general type of those defined by difference equations in accordance with the 
theorem quoted in section 7 , namely,

$$
g(x) \sim x^{-(s+1) x+\mu} e^{\alpha x}\left(1+\frac{c_{1}}{x}+\frac{c_{2}}{x^{2}}+\cdots\right),
$$

it is easy to see that $g(x+n) / g(x)$ has an asymptotic representation of the form

$$
\frac{g(x+n)}{g(x)} \sim x^{-(s+1) n}\left(\beta_{0 n}+\frac{\beta_{1 n}}{x}+\frac{\beta_{2 n}}{x^{2}}+\cdots\right) .
$$

If we take for the value of $s$ a non-negative integer this function $g(x)$ gives rise to the $T$-series

$$
T(x)=P_{0_{\nu}}(x)+\sum_{n=1}^{\infty} P_{n \nu}(x) \frac{g\left(x^{t}+n\right)}{g\left(x^{t}\right)},
$$

where

$$
P_{n \nu}(x)=c_{n 0}+\frac{c_{n 1}}{x}+\frac{c_{n 2}}{x^{2}}+\cdots+\frac{c_{n \nu}}{x^{\nu}} \quad(n=0,1,2, \cdots) .
$$

If we take $\nu=s t+t-1$ it is easy to see that this $T$-series may be formally transformed uniquely into a descending power series

$$
\beta_{0}+\frac{\beta_{1}}{x}+\frac{\beta_{2}}{x^{2}}+\cdots,
$$

and conversely that any such descending power series may be formally transformed uniquely into a series $T(x)$ defined by means of any basic function $g(x)$ having the asymptotic character (9).

A special case of the foregoing series of particular interest is that depending on a function $g(x)$ for which in (9) we have $\mu=\alpha=0=c_{1}=c_{2}=c_{3}=\cdots$.

It may be added that the function $T(x)$ defined by (10) is asymptotic to the power series into which the series $T(x)$ is transformable; a proof will be given in a later paper.

\section{Character of the Regions of Convergence.}

19. In my Transactions paper referred to in $\S 3$ above I have proved for the general series $\Omega(x)$ in (3) the following theorem:

If $\sigma$ denotes $\alpha_{m}$ or $\mu_{k}$ according as $m$ is or is not greater than 
$k$, then there exists a unique real number $\lambda[\mu]$ such that the region of convergence (absolute convergence) (exclusive of the exceptional points) of the series $\Omega(x)$ is bounded by the straight line $R(\sigma x)=\lambda[R(\sigma x)=\mu]$ and lies on that side of this line for which $R(\sigma x)<\lambda[R(\sigma x)<\mu]$.

We shall now extend this theorem to the case of the series $T(x)$.

In view of the theorem just quoted it is easy to see that the series

$$
\Omega_{i}\left(x^{t}\right)=\sum_{n=0}^{\infty} c_{n i} \frac{g\left(x^{t}+n\right)}{g\left(x^{t}\right)}
$$

has a region of convergence (absolute convergence) bounded by the curve

$$
R\left(\sigma x^{t}\right)=\lambda_{i} \quad\left[R\left(\sigma x^{t}\right)=\mu_{i}\right],
$$

where $\lambda_{i}\left[\mu_{i}\right]$ is an appropriately determined constant, and that it lies on that side of this curve for which

$$
R\left(\sigma x^{t}\right)<\lambda_{i} \quad\left[R\left(\sigma x^{t}\right)<\mu_{i}\right] .
$$

Let us now consider the $\nu+1$ series (11) for which $i$ takes the values $0,1,2, \cdots, \nu$. Multiplying $\Omega_{i}\left(x^{t}\right)$ by $1 / x^{i}(i=0$, $1, \cdots, \nu)$ and adding the resulting series term by term we have

$$
T_{\nu t}(x)=\sum_{n=0}^{\infty} P_{n \nu}(x) \frac{g\left(x^{t}+n\right)}{g\left(x^{t}\right)},
$$

where

$$
P_{n \nu}(x)=c_{n 0}+\frac{c_{n 1}}{x}+\cdots+\frac{c_{n \nu}}{x^{\nu}} .
$$

Let $\rho[\tau]$ be a number of the set $0,1,2, \cdots, \nu$ such that $\lambda_{\rho} \leqq \lambda_{i}, i=0,1, \cdots, \nu, i \neq \rho\left[\mu_{\tau} \leqq \mu_{i}, i=0,1, \cdots, \nu, i \neq \tau\right]$. Then it is clear that $T_{v t}(x)$ converges (converges absolutely) for any value of $x$ such that

$$
R\left(\sigma x^{t}\right)<\lambda_{\rho} \quad\left[R\left(\sigma x^{t}\right)<\mu_{\tau}\right] .
$$

If furthermore $\lambda_{\rho}<\lambda_{i}$ when $i \neq \rho$, then the region of convergence of $T_{v t}(x)$ is bounded by the curve

$$
R\left(\sigma x^{t}\right)=\lambda_{\rho} .
$$

If on the other hand $\lambda_{\rho}\left[\mu_{\tau}\right]$ is equal to some other $\lambda[\mu]$, then 
$T_{\nu t}(x)$ may converge (converge absolutely) for certain nonexceptional values of $x$ outside of the region defined by (13). This is put in evidence by the series

$$
\sum_{n=0}^{\infty}\left[\left(\frac{1}{n !}+2\right)+x\right] \frac{\Gamma(x) \Gamma(n)}{\Gamma(x+n)} .
$$

Here $\lambda_{0}=\mu_{0}=\lambda_{1}=\mu_{1}=1$, as one may show without great difficulty by making use of the asymptotic character of $\Gamma(x)$. This series converges absolutely for the non-exceptional value $x=-2$ although both relations in (13) now reduce to $R(-x)<1$ or $R(x)>-1$.

In view of this possibility that $T_{v t}(x)$ may converge (converge absolutely) outside of the common part of the regions of convergence (absolute convergence) of the component series (11) it seems desirable to state the following theorem, the proof of which is immediate in view of relation (10) in my Transactions paper already referred to:

Let $x_{0}$ and $x_{1}$ be two values of $x$ which are non-exceptional for the series $T_{\nu t}(x)$ and suppose that $T_{\nu t}\left(x_{0}\right)$ converges absolutely. Then $T_{v t}\left(x_{1}\right)$ converges absolutely if $R\left(\sigma x_{1}^{t}\right)<R\left(\sigma x_{0}{ }^{t}\right)$ and the quotient

is bounded.

$$
P_{n \nu}\left(x_{1}\right) / P_{n \nu}\left(x_{0}\right)
$$

20. We should examine briefly the nature of the curves

$$
R\left(\sigma x^{t}\right)=\eta
$$

which bound the regions of convergence and absolute convergence of series $T_{v t}(x)$. For this purpose we write

$$
\sigma=|\sigma| e^{\phi \gamma \overline{-1}}, \quad x=r e^{\theta V \overline{-1}},
$$

where $r$ is real and not negative. Then (14) reduces readily to the relation

$$
r^{t} \cos (t \theta+\varphi)=\eta_{1}
$$

where

$$
\eta_{1}=\eta /|\sigma| \text {. }
$$

In case $\eta_{1}=0$ equation (15) represents $2 t$ rays from zero to infinity and dividing the space about zero into $2 t$ equal parts or sectors. The quantity denoted by the first member of (15) is negative within alternate sectors of this set (the sectors of convergence) and positive within the others (in 
general the sectors of divergence). If $\eta_{1}$ is negative then (15) represents a curve of $t$ branches lying within the aforementioned sectors within which the first member of (15) denotes a negative quantity; and each branch approaches asymptotically the rays of the sector including it. In this case the region of convergence consists in general of $t$ separated regions each bounded by a curve somewhat resembling one branch of an hyperbola. In case $\eta_{1}$ is positive the branches of the curve denoted by (15) lie in the aforementioned sectors within which the first member of (15) is positive; and again each branch approaches asymptotically the rays of the sector including it. In this case the region of convergence is in general the portion of the plane excluded by the $t$ branches, which again resemble branches of hyperbolas.

UNIVERSITY OF ILLINOIS, April 14, 1917.

\section{SHORTER NOTICE.}

Interpolated Six-place Tables. Edited by Horace Wilmen Marsh. New York, John Wiley and Sons, 1916. xii + $155 \mathrm{pp}$.

THIs volume contains logarithms of numbers and the natural and logarithmic trigonometric functions, also tables of length, area, volume, weight, metric conversion, decimal equivalents, and specific gravity. In view of the many logarithmic tables on the market, a new compilation is expected to present valuable improvements. Only one such is evident in this book, viz., the use of a heavy ruling to denote "the change in leading figures when occurring in the line, thereby making the use of the wrong leading figures possible only by "jumping the fence." Unfortunately on page 39 , an omitted "fence" gives wrong values for $\log \tan 3^{\circ} 0^{\prime}, 20^{\prime \prime}, 30^{\prime \prime}, 40^{\prime \prime}$, and $50^{\prime \prime}$.

In the logarithms of numbers no horizontal spacings or rulings are used and the number of rows on a page varies from 13 to 31 , so that the position of the desired logarithm on the page is never known "a priori." In the logarithmic trigonometric tables the lines are separated into groups of ten for 6 pages only, though this grouping is used throughout the 